\title{
Simulated Photo-nuclear Kaon Production for LDMX
}

\section{Chloe Greenstein - Lewis \& Clark College - Cristina Mantilla Suárez, FNAL - FERMILAB-POSTER-21-054-STUDENT}

\section{Light Dark Matter eXperiment (LDMX)}

The Light Dark Matter Experiment, LDMX, is a proposed experiment for detecting sub-GeV dark matter. LDMX looks for dark matter produced in an electron fixed target setup through the recreation of primordial interactions between dark matter and Standard Model Particles.

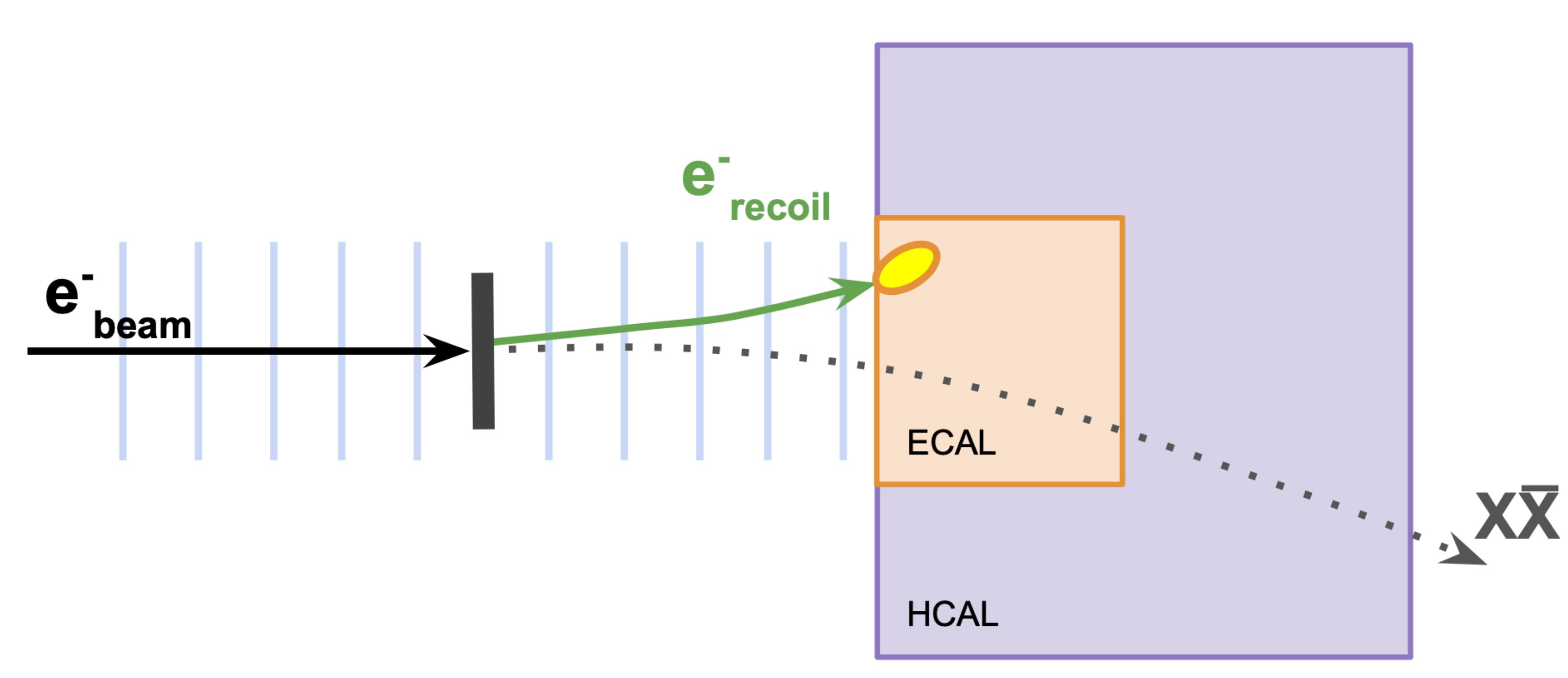

Fig. 1: Conceptual drawing of the LDMX experiment, showing the electron beam passing through a tagging tracker, a thin tungsten target, the recoil tracker, the electromagnetic calorimet

\section{Goal: K-long background rate}

Photo-nuclear events, originated when a Bremsstrahlung photon interacts with the target, can produce hadrons that, if undetected, mimic our dark matter signal. Neutral kaons (K-short) that decay into charged pions are reconstructible in the ECAL, but those that decay into neutrinos (K-long or $\mathrm{K}+$ ) can go undetected. Understanding the yields and kinematics in K-short decay allows to understand the rate of the neutrino background.

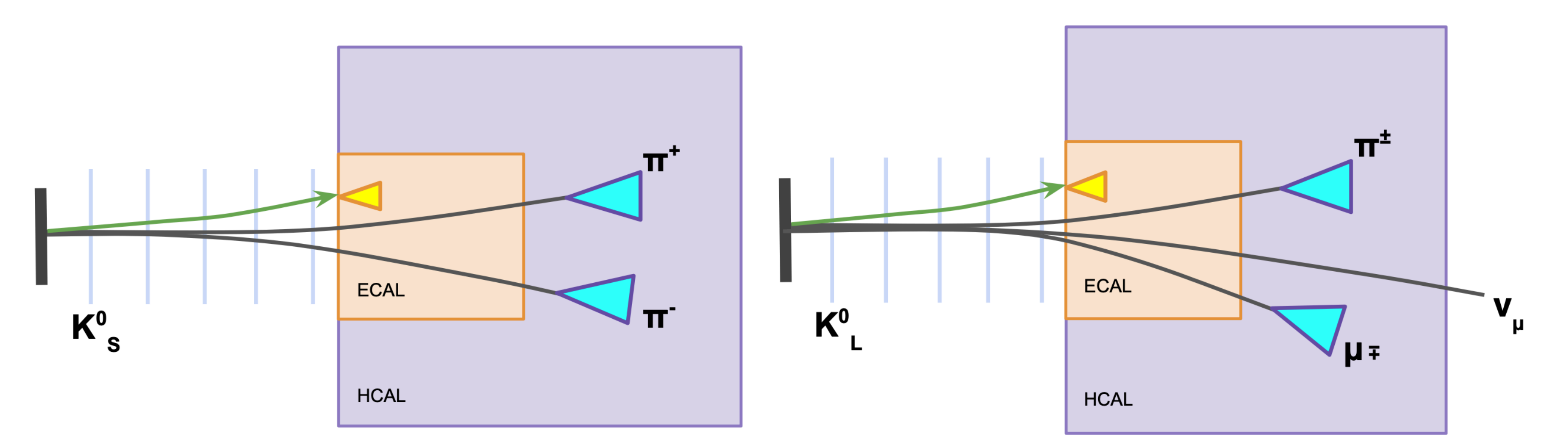
Fig. 2: Drawing of the LDMX experiment, showing the decay of K-short (left) and K-
long(right) traveling from the target, through the tagging tracker, ECAL, HCAL, and where the products are detected in the ECAL and HCAL.

\section{Simulated event generation}

- Photo-nuclear events with $4 \mathrm{GeV}$ incident electron - Events have a Bremsstrahlung process in the target region with a photon energy $>2.5 \mathrm{GeV}$ (electron recoil $<1.5 \mathrm{GeV}$ )

- Arbitrary large bias factor (1e9) to artificially increase background rate

- Biased Event Rate:

- 1 Truth Ks per 48 Events Generated

- Non Biased Event Rate:

- 1 Truth Ks per 50,000 Events Generated

- Ks rate with 1e16 EOT: $\sim \mathbf{3 . 4 e 6} \mathrm{Ks}$

\section{Reconstructing K-short}

We only consider particles with an angle less than 40 degrees from the beam line. This mask was applied to all daughters from the Kaon decay. We reconstruct the Kaon mass by looking at the daughters from simulation. We smear the momentum in the $x, y$, and $z$ dimensions with the expected track resolution. We can reconstruct the mass with $20 \mathrm{GeV}$ resolution.
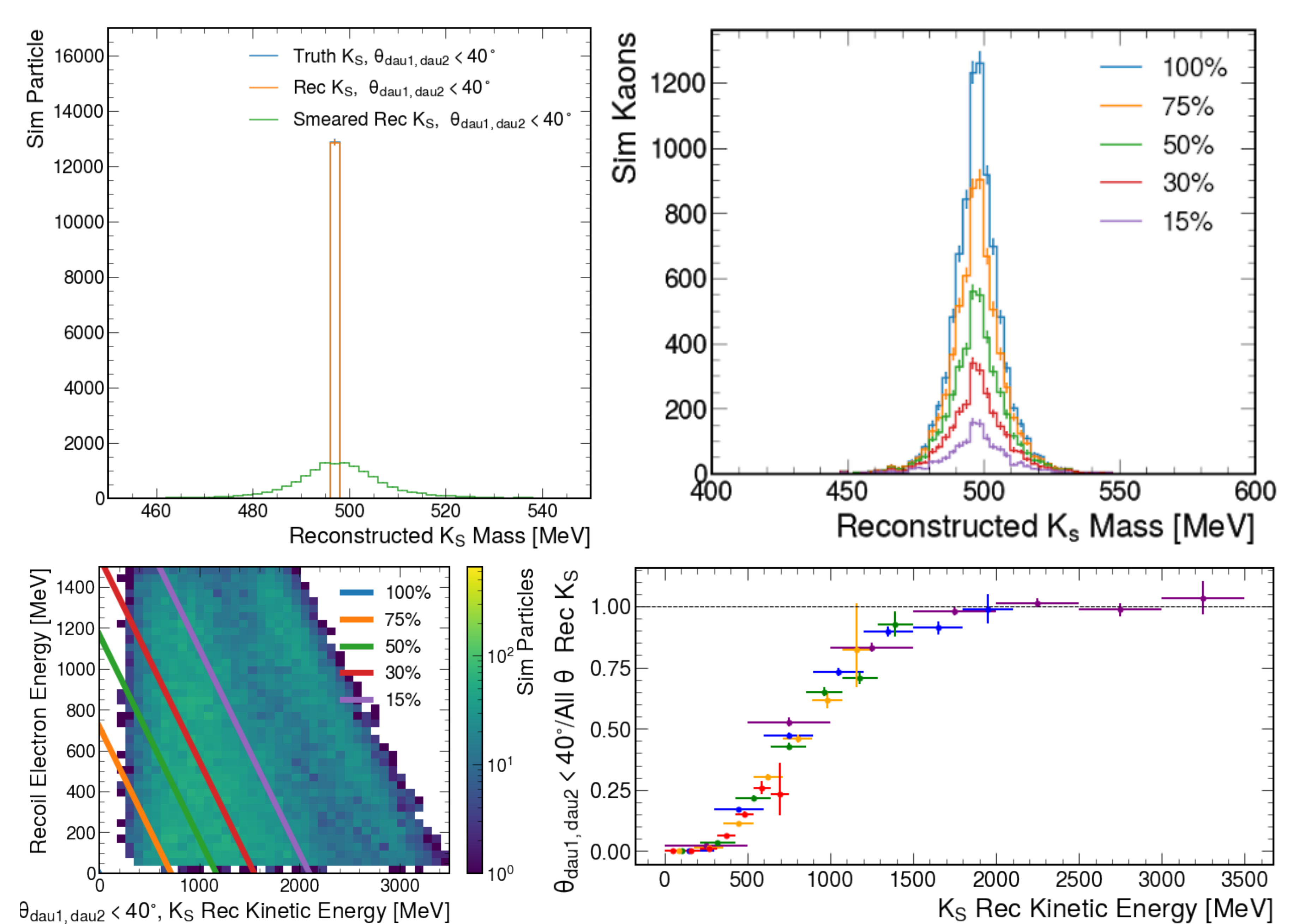
$\vartheta_{\mathrm{d} d u 1, \text { dau2 }} 40^{\circ}, \mathrm{K}_{\mathrm{S}}$ Rec Kinetic Energy [MeV]

Fig. 3: (Top Left) Reconstructed K-short mass compared to truth values of the Kaon mass. (Top Right) Reconstructed K-short mass by percentile included from the most energetic.(Bottom Left) Kinetic energy of reconstructed Kaons vs. recoil electron and their percentile sectioning. (Bottom Right) ratio of all reconst
reconstructed with theta cut, separated by energy percentile.
Truth $\mathrm{K}_{\mathrm{L}} / \mathrm{K}_{\mathrm{s}}$ Ratio
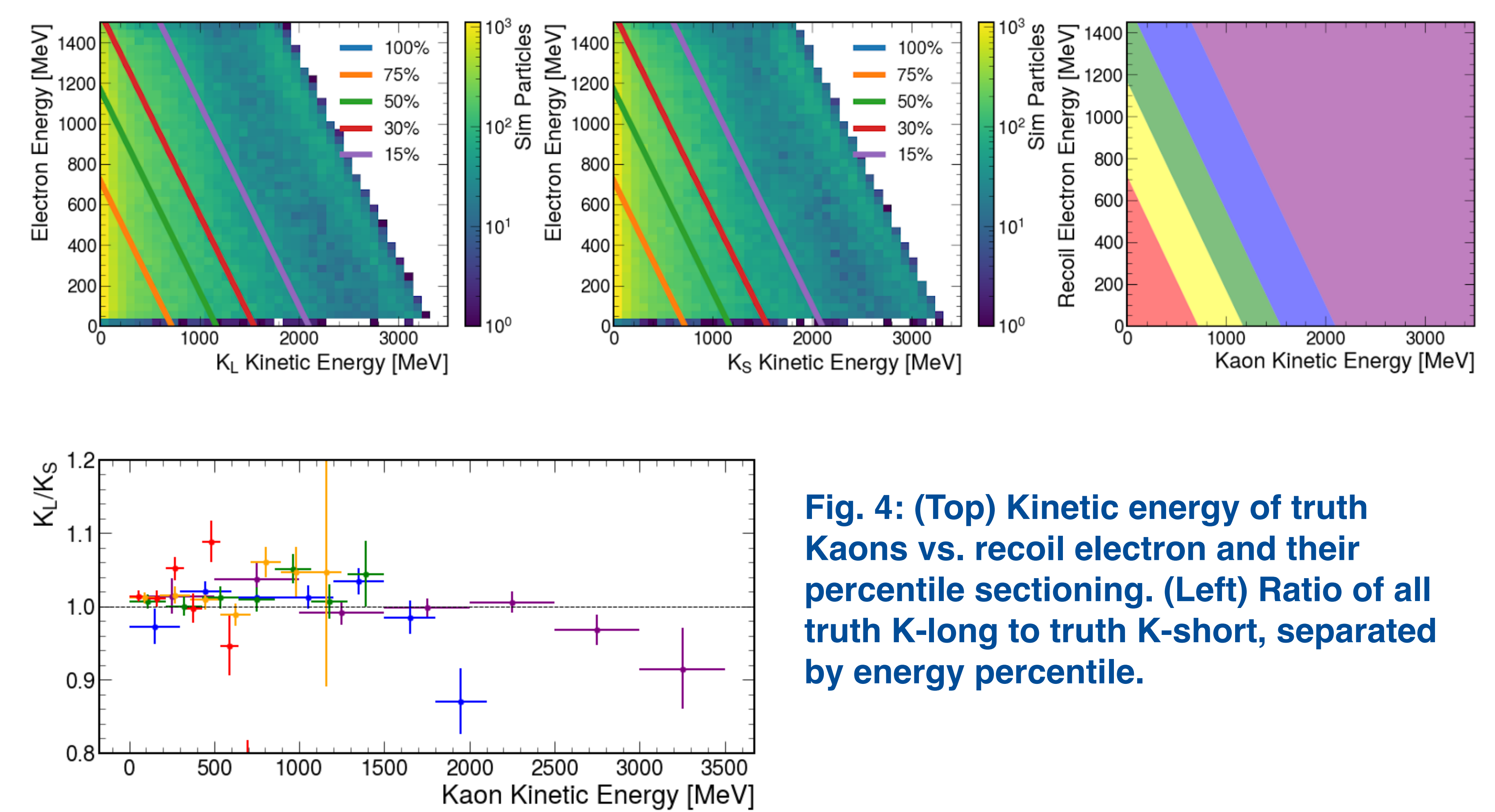

Fig. 4: (Top) Kinetic energy of truth Kaons vs. recoil electron and their truth K-long to truth K-short, separated by energy percentile.

In multiple types of events and different energy levels, the ratio of truth $\mathrm{K}$-long to $\mathrm{K}$-short remains around one. This is hopeful in that K-short can be used to account for the semi-visible decays of $\mathrm{K}$-long.

\section{Reconstructing K-long from K-short}

The branching ratio acceptance and these ratios can reconstruct $\mathrm{K}$-long by the following:

$$
K_{L}=\frac{K_{L}}{K_{S}} * \frac{K_{S}\left(\pi^{+} \pi^{-}: \text {measured }\right)}{B R_{\pi^{+}} \pi^{-}}
$$

These studies determined visible PN decays of K-short can successfully reconstruct the semi-visible PN decays of K-long.

\section{Acknowledgements}

Thank you to the LDMX team at Fermilab National Accelerator Laboratory for the opportunity and collaboration. Thank you especially to this project's supervisor, Cristina Mantilla Suárez, for her expertise and guidance. Thank you, Nhan Tran and Christian Herwig, for your advice and encouragement in the LDMX project

This manuscript has been authored by Fermi Research Alliance, LLC under Contract No. DE-AC02-07CH11359 with the U.S. Department of Energy, Office of Science, Office of High Energy Physics. 